\title{
Impact of bleeding-related complications and/or blood product transfusions on hospital costs in inpatient surgical patients
}

Michael E Stokes ${ }^{1 *}$, Xin Ye ${ }^{2}$, Manan Shah ${ }^{3}$, Katie Mercaldi ${ }^{4}$, Matthew W Reynolds ${ }^{4}$, Marcia FT Rupnow ${ }^{2}$ and Jeffrey Hammond ${ }^{2}$

\begin{abstract}
Background: Inadequate surgical hemostasis may lead to transfusion and/or other bleeding-related complications. This study examines the incidence and costs of bleeding-related complications and/or blood product transfusions occurring as a consequence of surgery in various inpatient surgical cohorts.

Methods: A retrospective analysis was conducted using Premier's Perspective ${ }^{\mathrm{TM}}$ hospital database. Patients who had an inpatient procedure within a specialty of interest (cardiac, vascular, non-cardiac thoracic, solid organ, general, reproductive organ, knee/hip replacement, or spinal surgery) during 2006-2007 were identified. For each specialty, the rate of bleeding-related complications (including bleeding event, intervention to control for bleeding, and blood product transfusions) was examined, and hospital costs and length of stay (LOS) were compared between surgeries with and without bleeding-related complications. Incremental costs and ratios of average total hospital costs for patients with bleeding-related complications vs. those without complications were estimated using ordinary least squares (OLS) regression, adjusting for demographics, hospital characteristics, and other baseline characteristics. Models using generalized estimating equations (GEE) were also used to measure the impact of bleeding-related complications on costs while accounting for the effects related to the clustering of patients receiving care from the same hospitals.

Results: A total of 103,829 cardiac, 216,199 vascular, 142,562 non-cardiac thoracic, 45,687 solid organ, 362,512 general, 384,132 reproductive organ, 246,815 knee/hip replacement, and 107,187 spinal surgeries were identified. Overall, the rate of bleeding-related complications was $29.9 \%$ and ranged from $7.5 \%$ to $47.4 \%$ for reproductive organ and cardiac, respectively. Overall, incremental LOS associated with bleeding-related complications or transfusions (unadjusted for covariates) was 6.0 days and ranged from 1.3 to 9.6 days for knee/hip replacement and non-cardiac thoracic, respectively. The incremental cost per hospitalization associated with bleeding-related complications and adjusted for covariates was highest for spinal surgery $(\$ 17,279)$ followed by vascular $(\$ 15,123)$, solid organ $(\$ 13,210)$, non-cardiac thoracic $(\$ 13,473)$, cardiac $(\$ 10,279)$, general $(\$ 4,354)$, knee/hip replacement $(\$ 3,005)$, and reproductive organ $(\$ 2,805)$.

Conclusions: This study characterizes the increased hospital LOS and cost associated with bleeding-related complications and/or transfusions occurring as a consequence of surgery, and supports implementation of bloodconservation strategies.
\end{abstract}

\footnotetext{
* Correspondence: michael.stokes@unitedbiosource.com

'United BioSource Corporation, Montréal, QC, Canada

Full list of author information is available at the end of the article
} 


\section{Background}

Bleeding can be a complication of surgery that may lead to substantial morbidity and mortality. In cardiac surgery, severe bleeding occurs in approximately $7 \%$ of cases [1] and is associated with an increased risk of post-operative mortality [2]. Mortality rates approaching 20\% have been observed in elective vascular patients with severe bleeding complications [3]. In trauma patients, uncontrolled bleeding is the cause of between $30 \%-40 \%$ of all traumarelated deaths [4]. Bleeding complications may also occur from percutaneous procedures that are not considered surgical [5]. Approximately $7 \%$ of percutaneous coronary interventions (PCI) performed emergently in patients with acute coronary syndrome result in major bleeding [6]. As with procedures classified as surgical, severe bleeding from PCI has also been linked to significant increases in morbidity and mortality [7].

Blood transfusions, which can be planned/expected or unexpected, and reoperations are used to control bleeding and avert deaths. These procedures are often costly and require large amounts of clinical and staff resources [1]. Blood transfusions are not without considerable risks including nosocomial infection [8,9], immunosuppression [10], transfusion-related acute lung injury [11], and even death $[12,13]$. These complications may add additional costs to the hospitalization and even postdischarge medical care [1].

The purpose of this study was to estimate the economic burden associated with bleeding-related complications and/or transfusions occurring as a consequence of surgery in eight inpatient surgical cohorts, including cardiac, vascular, non-cardiac thoracic, solid organ, general surgery, knee/hip replacement, reproductive organ, and spinal surgery. These procedures represent the majority of all inpatient surgical procedures performed in the United States (US) [14], and inpatient data on the costs of treating patients with bleeding-related complications in these procedure groups are limited.

\section{Methods}

\section{Data Source}

Data for this retrospective cohort study were obtained from Premier's Perspective ${ }^{\mathrm{TM}}$ Comparative Database (PCD)[15]. The current study used PCD data containing inpatient, clinical, drug utilization, and hospital billing data from more than 600 hospitals throughout the US. Premier collects data from participating hospitals in its healthcare alliance. The Premier healthcare alliance was formed for hospitals to share knowledge, improve patient safety, and to reduce risks. Participation in the Premier healthcare alliance is voluntary. The PCD is comprised of hospital administrative data from the United States and, although the PCD excludes federally- funded hospitals (e.g., Veterans Affairs), the hospitals included are nationally representative based on bed size, geographic location, and teaching hospital status [16]. Approximately 5 million new hospital discharges are added to the database each year. According to the Healthcare Utilization Project (HCUP) data, there were 39.5 million hospital discharges throughout the US in 2007 [17]. Therefore, the Premier data used at the time of this analysis represented approximately $13 \%$ of all US hospital discharges. All hospitals participating in the healthcare alliance submit data on all patients, payors, and providers as captured on the hospital billing record to Premier. The data go through quality assurance and validation checks and once the data have been validated the information is added to the database. The PCD contains a patient-level date-stamped log for all procedures, medications, laboratory, and diagnostic services rendered during the hospital stay. Data elements include hospital and patient identifiers, primary and secondary ICD-9-CM diagnosis and procedure codes, length of hospital stay, admission type, and primary payer. Also available are data elements for demographic and hospital characteristics, including age, race, geographic location of provider, hospital bed size, teaching hospital status, and hospital location (urban or rural). Hospitals submit demographic data including age and patient reported race (White, Black, Hispanic, American Indian, Asian/ Pacific Islander, and Other). Data on hospital characteristics including hospital bed size, teaching hospital status (yes/no), and hospital location (urban/rural) are reported by hospitals. The geographic location of the provider (New England, Middle Atlantic, East North Central, West North Central, South Atlantic, East South Central, West South Central, Mountain, and Pacific) is based on Centers for Medicare and Medicaid Services metropolitan statistical area regions. Patient and provider information contained within the PCD are de-identified making it fully compliant with the Health Insurance Portability and Accountability Act privacy regulations. Institutional review board (IRB) approval for this study was not required as dictated by Title 45 CFR (Code of Federal Regulations) Part 46 of the United States under the exemption that this research involved the study of existing data and that the information was recorded in such a manner that the subjects could not be identified, directly or through identifiers linked to the subjects [18]. Hence, IRB approval was not obtained.

\section{Patient Population}

All patients in the PCD undergoing an inpatient surgery of interest (cardiac, vascular, non-cardiac thoracic, solid organ, general, knee/hip replacement, reproductive organ, and spinal surgery) from January 1, 2006 to 
December 31, 2007 were selected for inclusion. Patients of all ages were included in this study. Procedures related to surgeries of interest were identified using the International Classification of Diseases, Ninth RevisionClinical Modification (ICD-9-CM) procedure codes (Table 1). For patients with multiple hospitalizations, the most recent hospitalization (i.e., index) was selected for analysis to maximize the pre-index study period available for an assessment of baseline clinical and demographic characteristics. Patients were excluded if they were transferred from another hospital or an unknown source to ensure that complete hospitalization data were available for every patient. Patients were then classified into study subgroups by procedure type (cardiac, vascular, non-cardiac thoracic, solid organ, general surgery, knee/hip replacement, reproductive organ, and spinal surgery). A minority of patients (approximately $5 \%$ ) was included in $>1$ study subgroup because they were operated on in surgical sites spanning multiple surgical categories during the index hospitalization. For example, patients who had an operation on the vessels of their head and neck in addition to their heart valves would have been included in the separate vascular and cardiac cohort analyses, respectively.

\section{Bleeding-related Complication and/or Blood Product Transfusion status}

Within each procedure cohort, patients were classified into exposure groups according to whether a bleedingrelated complication and/or blood product transfusion occurred during the hospital stay. Bleeding-related complications and/or blood product transfusions were identified if the hospital record contained any of the following codes: ICD-9-CM diagnosis codes for hemorrhages or hematomas complicating a procedure (998.11 and 998.12); ICD-9-CM procedure codes for interventions, including return to the operating room to control for bleeding $(34.09,21.0 \times, 42.33,45.43,44.44,44.49$, $54.19,39.41,60.94,06.02,28.7,34.03,54.12,49.95$, and 57.93); ICD-9-CM diagnosis codes for blood product

Table 1 ICD-9-CM Procedure Codes Used to Identify Surgical Subgroups

\begin{tabular}{|c|c|}
\hline Type of Surgery & ICD-9-CM Procedure Codes \\
\hline \multicolumn{2}{|l|}{ Cardiac } \\
\hline Heart Valves & 35.00-35.99 \\
\hline Heart Vessels & $36.03,36.10-36.2,36.91-36.99$ \\
\hline Other Operations on Heart and Pericardium & $37.10-37.12,37.31-37.54,37.62-37.68$ \\
\hline Miscellaneous & $39.0,39.61-39.64,39.66,33.6$ \\
\hline \multicolumn{2}{|l|}{ Vascular } \\
\hline Incision, Excision, and Occlusion of Vessels & $38.00-38.18,38.30-38.49,38.60-38.89$ \\
\hline Other Operations on Vessels & 39.21-39.41, 39.49 \\
\hline \multicolumn{2}{|l|}{ Non-cardiac Thoracic } \\
\hline Lung and Bronchus & $32.01-32.21,32.24,32.25,32.28-32.9$ \\
\hline Other Operations on Lung and Bronchus & 33.0-33.52, 33.91-33.99 \\
\hline Chest Wall, Pleura, Mediastinum, and Diaphragm & $34.01-34.21,34.24-34.27,34.29-34.59,34.72-34.89,34.93-34.99$ \\
\hline Esophagus & $42.01,42.09,42.10-42.12,42.19,42.40-42.42,42.51-42.59,42.61-42.69,42.7$ \\
\hline \multicolumn{2}{|l|}{ General } \\
\hline Stomach & $43.0,43.19-43.3,43.42-44.11,44.15,44.21,44.29-44.43,44.49-44.91,44.95-44.99$ \\
\hline Intestine & $45.11,45.13-45.15,45.19,45.26-45.41,45.49-46.99$ \\
\hline Gall Bladder & $51.04,51.13,51.22-51.83,51.89-51.94$ \\
\hline \multicolumn{2}{|l|}{ Solid Organ } \\
\hline Pancreas & $52.01-52.09,52.12,52.21-52.96,52.99$ \\
\hline Kidney & $55.11,55.12,55.24,55.31-55.91,55.95-55.99$ \\
\hline Liver & $50.0,50.12-50.99$ \\
\hline Spleen & $41.1,41.2,41.41-41.5,41.93-41.99$ \\
\hline \multicolumn{2}{|l|}{ Knee/Hip Replacement } \\
\hline Knee & $81.54,00.80-00.83$ \\
\hline Hip & $80.05,81.51,81.52,81.53,00.70-00.73,00.85-00.87$ \\
\hline \multicolumn{2}{|l|}{ Reproductive Organ } \\
\hline Male & $60.0-63.71,63.81-63.99,64.11,64.2-64.3,64.42-64.45,64.92,64.93,64.95-64.99$ \\
\hline Female & $65.01-66.11,66.2-69.6,69.91,69.93-69.99,70.12-70.14,70.23-70.29,70.32-71.11,71.3-71.9$ \\
\hline Spinal Surgery & $03.2 \times, 03.4 \times, 03.5 \times, 03.6 x, 84.6 x, 84.8 x, 81.0 \times, 81.3 x, 81.62-81.64$ \\
\hline
\end{tabular}

ICD-9-CM = The International Classification of Diseases, Clinical Modification, Ninth Revision 
transfusions (V58.2, E879.8, E873.0, E934.7, and E876.0); or ICD-9-CM procedure codes for blood product transfusions (99.00-99.09).

\section{Study Outcomes}

The outcomes measured in this study included the total length of stay (LOS) in days, the number of days spent in an intensive care unit (ICU), and total hospital costs. Total hospital costs were the actual treatment costs incurred by the hospital and were the sum of the hospital's direct and overhead costs. Direct costs represented services that were used by the patient and included items such as the costs of physicians employed directly by the hospital, treatment costs, and food [19]. Direct costs are recorded in the hospital accounting system as they get itemized for each patient and represent the costs of providing care from the hospital's perspective. Cost variables are not tied to reimbursement or payments from health insurers. Overhead costs were costs associated with the overall functioning of the hospital and included nursing, administrative, and management staffing costs, as well as electricity and the depreciation of medical equipment [19]. These costs are added to each patient record in the hospital accounting system as a standard cost according to the hospital department(s) providing care. Costs accrued during the 2006 study calendar year were standardized to 2007 \$US using the medical care component of the Consumer Price Index.

\section{Data Analyses}

The proportion of patients experiencing a particular type of complication was assessed according to surgical subgroup. Baseline demographic, clinical, and hospital characteristics were evaluated according to bleedingrelated complication and/or blood product transfusion status for all surgical subgroups combined. Pre-operative use of substances that promote hemostasis was defined as receipt of a hemostat in any of the hemostat classes including oxidized regenerated cellulose, collagen, gelatin, fibrin sealant, thrombin, flowables, combination products, and adhesion prevention. Group comparisons were made between patients with and without complications using 2-sided Pearson chi square and $t$-test statistics for categorical and continuous measures, respectively. Statistical significance was evaluated at alpha $=0.05$.

The total cost of hospitalization was analyzed for patients with bleeding-related complications and/or blood product transfusions versus those without a bleeding-related complication or transfusion. Patients with hospital costs exceeding $\$ 1,000,000$ were excluded from the analysis of costs. Multivariate ordinary least squares regression models were created for each surgical population to measure the impact of bleeding-related complications and/or blood product transfusion events on total hospital costs after controlling for important baseline and clinical patient characteristics. As cost data generally do not follow a normal distribution and are often right-skewed, total costs were transformed to their natural logs. Adjusted mean log costs were then retransformed using Duan's smearing estimate [20]. Baseline parameters, clinical characteristics, and comorbid conditions deemed to have an impact on total hospital costs were considered for inclusion into multivariate models, including bleeding-related complication and/or blood product transfusion status, age, gender, race, geographic region, hospital location, year of surgery, teaching hospital status, hospital bed size, surgical admission type (urgent, elective, or emergency), any cause hospitalization within 6 months prior to the index hospitalization, surgery at multiple sites, and comorbid conditions as independent variables. Comorbid conditions included thrombocytopenia, myocardial infarction (MI), hypertension, non-MI coronary disease, diabetes, peripheral vascular disease, obesity, chronic obstructive pulmonary disease (COPD), renal disease, congestive heart failure, cancer, deep vein thrombosis, and liver cirrhosis. All comorbidities were modeled as separate covariates. Statistical comparisons across groups were conducted using a t-test evaluating the null hypothesis that the parameter estimate for bleeding-related complications and/or blood product transfusions is equal to 0 . Statistical significance was evaluated at alpha $=0.05$. The incremental difference in adjusted mean hospital costs between patients with a bleeding event and/or blood product transfusion and those without these events was assumed to be the hospital costs attributable to having a bleeding event and/or blood product transfusion. Generalized estimating equation (GEE) models with a Gamma distribution and log link function were also used to measure the impact of bleeding-related complications and/or blood product transfusion events on total hospital costs while accounting for the effects related to the clustering of patients receiving care from the same hospitals. The same baseline parameters used for the ordinary least squares regression models of total hospital costs were included in GEE models.

\section{Results}

Table 2 displays the numbers of patients who developed a bleeding-related complication and/or received a blood product transfusion during the surgical hospitalization. Results are displayed by complication type and surgical subgroup. The percentage of patients with any type of bleeding-related complication varied according to subgroup (general: 27.5\%; cardiac: $47.4 \%$; solid organ: 28.5\%; non-cardiac thoracic: $34.3 \%$; vascular: $31.5 \%$; knee/hip replacement: $29.8 \%$; reproductive organ: $7.5 \%$; 
Table 2 Percentages of Patients with Specific Complication Events, by Surgical Subgroup

\begin{tabular}{|c|c|c|c|c|c|c|c|c|}
\hline Complication & Cardiac & Vascular & $\begin{array}{l}\text { Non-cardiac } \\
\text { Thoracic }\end{array}$ & $\begin{array}{l}\text { Solid } \\
\text { Organ }\end{array}$ & General & $\begin{array}{l}\text { Reproductive } \\
\text { Organ }\end{array}$ & $\begin{array}{c}\text { Knee/Hip } \\
\text { Replacement }\end{array}$ & Spinal \\
\hline Total number of patients & 103,829 & 216,199 & 142,562 & 45,687 & 362,512 & 384,132 & 246,815 & 107,187 \\
\hline Bleeding event only, \% & $0.9 \%$ & $1.9 \%$ & $0.7 \%$ & $1.0 \%$ & $0.5 \%$ & $0.8 \%$ & $0.5 \%$ & $0.5 \%$ \\
\hline Re-operation to control bleeding only, \% & $0.4 \%$ & $0.4 \%$ & $4.7 \%$ & $0.6 \%$ & $1.9 \%$ & $0.2 \%$ & $0.0 \%$ & $0.2 \%$ \\
\hline Blood product transfusion only, $\%$ & $40.9 \%$ & $25.2 \%$ & $22.7 \%$ & $22.8 \%$ & $19.9 \%$ & $5.7 \%$ & $28.6 \%$ & $13.6 \%$ \\
\hline Bleeding event and blood product transfusion, \% & $2.5 \%$ & $2.4 \%$ & $1.2 \%$ & $1.8 \%$ & $0.7 \%$ & $0.5 \%$ & $0.7 \%$ & $0.6 \%$ \\
\hline $\begin{array}{l}\text { Bleeding event and re-operation to control } \\
\text { bleeding, } \%\end{array}$ & $0.3 \%$ & $0.1 \%$ & $0.4 \%$ & $0.1 \%$ & $0.1 \%$ & $0.1 \%$ & $0.0 \%$ & $0.0 \%$ \\
\hline $\begin{array}{l}\text { Re-operation to control bleeding and blood } \\
\text { product transfusion, } \%\end{array}$ & $1.0 \%$ & $1.1 \%$ & $3.3 \%$ & $1.5 \%$ & $4.1 \%$ & $0.1 \%$ & $0.0 \%$ & $0.1 \%$ \\
\hline $\begin{array}{l}\text { Bleeding event, re-operation to control bleeding } \\
\text { and blood product transfusion, } \%\end{array}$ & $1.4 \%$ & $0.4 \%$ & $1.3 \%$ & $0.7 \%$ & $0.3 \%$ & $0.1 \%$ & $0.0 \%$ & $0.0 \%$ \\
\hline Any bleeding-related consequences, $\%$ & $47.4 \%$ & $31.5 \%$ & $34.3 \%$ & $28.5 \%$ & $27.5 \%$ & $7.5 \%$ & $29.8 \%$ & $15.0 \%$ \\
\hline
\end{tabular}

spine: $15.0 \%$; and overall: $29.9 \%$ ). The most common type of event was blood product transfusion, which occurred in $21.2 \%$ of all patients (cardiac: $45.8 \%$; vascular: $29.1 \%$; non-cardiac thoracic: $28.5 \%$; solid organ: 26.8\%; general: 25.0\%; knee/hip replacement: $29.3 \%$; reproductive organ: $6.4 \%$; and spine: $14.3 \%)$. The percentages of patients who had an intervention to control for bleeding ranged from $0.0 \%-9.7 \%$ (cardiac: $3.1 \%$; vascular: $2.0 \%$; non-cardiac thoracic: $9.7 \%$; solid organ: 2.9\%; general: 6.4\%; knee/hip replacement: $0.0 \%$; reproductive organ: $0.5 \%$; and spine: $0.3 \%$ ). Overall, interventions for bleeding control were relatively uncommon, occurring in only $2.5 \%$ of all surgical patients.

Baseline demographic and clinical characteristics are presented in Table 3 according to bleeding-related complication and/or blood product transfusion status. All differences in baseline demographic and clinical measures were highly significant across study groups due to the large sample size. Patients less than 18 years of age were included in this study and represented $2.5 \%, 1.2 \%$, $4.6 \%, 3.7 \%, 2.6 \%, 0.03 \%, 0.9 \%$, and $2.2 \%$ of patients in the cardiac, vascular, non-cardiac thoracic, solid organ, general, knee-hip replacement, reproductive organ, and spinal surgery subgroups, respectively (data not shown). It was possible for some patients to be operated on in surgical sites spanning multiple surgical categories during the index hospitalization. These patients accounted for $15.5 \%, 15.1 \%, 20.2 \%, 41.8 \%, 11.3 \%, 1.0 \%, 3.2 \%$, and $5.1 \%$ of patients in the cardiac, vascular, non-cardiac thoracic, solid organ, general, knee-hip replacement, reproductive organ, and spinal surgery subgroups, respectively (data not shown).

The authors interpreted differences between groups to be important for age, gender, primary payer, having a prior surgery as well as comorbidities including diabetes, COPD, and cancer. Patients who had a bleeding-related complication were on average 8.9 years older compared to patients without a complication (mean age 64.0 years vs. 55.1, $P<0.001)$. There were also relevant differences across study groups with respect to the patients' primary payer of medical care. There was a higher percentage of patients experiencing a bleeding-related complication and/or blood product transfusion who received Medicare reimbursement versus patients without these events (57.7\% vs. $37.8 \%, P<0.001)$. A higher percentage of male patients had a bleeding-related complication versus those without a complication $(45.5 \%$ vs. $36.8 \%, P<0.001)$. Patients with bleeding-related complications and/or blood product transfusions were more likely to have diabetes (46.2\% vs. $26.1 \%, P<0.001)$, COPD (25.2\% vs. $18.7 \%, P<0.001)$, and cancer $(22.9 \%$ vs. $16.9 \%, P<0.001)$ compared to those without these events. We interpreted statistically significant differences with respect to demographic characteristics such as race and geographic region as unimportant. Additionally, differences in prior hospitalization, pre-operative use of substances promoting hemostasis, liver cirrhosis, and obesity were deemed unimportant.

Table 4 reports the characteristics of the hospital in which the surgery occurred. There were no relevant differences in hospital size, hospital location (urban vs. rural), or the proportion of patients treated in a teaching hospital across study groups. However, differences were statistically significant due to large sample sizes. The authors interpreted the differences between study subgroups to be unimportant.

Data on hospitalization lengths of stay (data presented in days and unadjusted for covariates) are presented in Figure 1 by surgical cohort and complication status. Patients with bleeding-related complications and/or blood product transfusions experienced a longer LOS compared to patients without bleeding-related complications and blood product transfusions (overall: 10.4 vs. 4.4 days; cardiac: 11.0 vs. 6.2 days; vascular: 15.2 vs. 5.9 days; solid organ: 13.4 vs. 5.3 days; non-cardiac thoracic: 18.7 vs. 9.1 days; general: 12.9 vs. 5.7 days; knee/hip 
Table 3 Baseline Demographics and Clinical Characteristics, by Bleeding-related Complication and/or Blood Product Transfusion Status

\begin{tabular}{|c|c|c|c|}
\hline Characteristic & Complication* & $\begin{array}{c}\text { No } \\
\text { Complication }\end{array}$ & $P$-value \\
\hline Number of patients & 351,065 & $1,175,208$ & \\
\hline Age, mean years (SD) & $64.0(17.9)$ & $55.1(18.7)$ & $<0.001$ \\
\hline Range & $1-89$ & $1-89$ & \\
\hline Male, N (\%) & $\begin{array}{l}159,893 \\
(45.5 \%)\end{array}$ & $432,211(36.8 \%)$ & $<0.001$ \\
\hline \multicolumn{4}{|l|}{ Race, N (\%) } \\
\hline White & $\begin{array}{l}231,146 \\
(65.8 \%)\end{array}$ & $770,920(65.6 \%)$ & $<0.001$ \\
\hline Black & 46,288 (13.2\%) & 143,269 (12.2\%) & \\
\hline Hispanic & $12,842(3.7 \%)$ & $58,358(5.0 \%)$ & \\
\hline Other & 60,789 (17.3\%) & 202,661 (17.2\%) & \\
\hline \multicolumn{4}{|l|}{$\begin{array}{l}\text { Geographic Region, } \\
\text { N (\%) }\end{array}$} \\
\hline Northeast & 57,899 (16.5\%) & 163,160 (13.9\%) & $<0.001$ \\
\hline South & $\begin{array}{l}170,133 \\
(48.5 \%)\end{array}$ & $537,047(45.7 \%)$ & \\
\hline Midwest & $60,598(17.3 \%)$ & 259,391 (22.1\%) & \\
\hline West & $58,970(16.8 \%)$ & 204,439 (17.4\%) & \\
\hline Unknown & $3,465(1.0 \%)$ & $11,171(1.0 \%)$ & \\
\hline \multicolumn{4}{|l|}{ Primary Payer, N (\%) } \\
\hline Medicare & $\begin{array}{l}202,495 \\
(57.7 \%)\end{array}$ & 443,685 (37.8\%) & $<0.001$ \\
\hline Medicaid & 24,477 (7.0\%) & 110,654 (9.4\%) & \\
\hline Private & 98,132 (28.0\%) & $524,710(44.6 \%)$ & \\
\hline Uninsured & 13,495 (3.8\%) & $45,219(3.8 \%)$ & \\
\hline Other & $12,466(3.6 \%)$ & $50,940(4.3 \%)$ & \\
\hline \multicolumn{4}{|l|}{ Comorbidity, N (\%) } \\
\hline Diabetes & $\begin{array}{l}162,097 \\
(46.2 \%)\end{array}$ & $306,512(26.1 \%)$ & $<0.001$ \\
\hline Obesity & 41,646 (11.9\%) & $150,510(12.8 \%)$ & $<0.001$ \\
\hline COPD & 88,506 (25.2\%) & 219,635 (18.7\%) & $<0.001$ \\
\hline Cancer & 80,506 (22.9\%) & 198,206 (16.9\%) & $<0.001$ \\
\hline Cirrhosis & $11,034(3.1 \%)$ & $9,835(0.8 \%)$ & $<0.001$ \\
\hline $\begin{array}{l}\text { Prior Hospitalization, } \\
\text { N (\%) }\end{array}$ & $\begin{array}{l}161,956 \\
(46.1 \%)\end{array}$ & $486,348(41.4 \%)$ & $<0.001$ \\
\hline Prior Surgery, N (\%) & $\begin{array}{l}106,064 \\
(30.2 \%)\end{array}$ & $293,533(25.0 \%)$ & $<0.001$ \\
\hline $\begin{array}{l}\text { Pre-operative use of } \\
\text { substances that } \\
\text { promote hemostasis, } \\
\text { N (\%) }\end{array}$ & 7,957 (2.3\%) & $21,338(1.8 \%)$ & $<0.001$ \\
\hline
\end{tabular}

$\mathrm{N}$ = number of patients, $\mathrm{SD}=$ standard deviation

*Bleeding complication and/or blood product transfusion

replacement: 4.9 vs. 3.6 days; reproductive organ: 6.2 vs. 2.6 days; and spine: 7.8 vs. 3.3 days). Patients with bleeding-related complications and/or blood product transfusions in all surgical categories also spent more days in an ICU versus patients without bleeding-related complications and/or blood product transfusions (overall: 3.3 vs. 0.5 days; cardiac: 4.9 vs. 2.1 days; vascular: 6.0
Table 4 Hospital Characteristics, by Bleeding-related Complication and/or Blood Product Transfusion Status

\begin{tabular}{lcccc}
\hline Characteristic & Complication* & No Complication & $P$-value \\
\hline Number of patients & 351,065 & $1,175,208$ & \\
Hospital size-beds, N (\%) & & & \\
50-249 & $61,300(17.5 \%)$ & $219,235(18.7 \%)$ & $<0.001$ \\
$250-749$ & $250,774(71.4 \%)$ & $829,201(70.6 \%)$ & \\
$750+$ & $38,991(11.1 \%)$ & $126,772(10.8 \%)$ & \\
Teaching hospital, N (\%) & $153,739(43.8 \%)$ & $505,674(43.0 \%)$ & $<0.001$ \\
Hospital location, N (\%) & & & \\
$\quad$ Urban & $313,037(89.2 \%)$ & $1,043,060(88.8 \%)$ & $<0.001$ \\
$\quad$ Rural & $34,563(9.8 \%)$ & $120,977(10.3 \%)$ & \\
$\quad$ Unknown & $3,465(1.0 \%)$ & $11,171(1.0 \%)$ & \\
Hospital length of stay & & & \\
$\quad$ Mean (SD) & $10.4(13.5)$ & $4.5(5.4)$ & $<0.001$ \\
Median (Range) & $6.0(1-615)$ & $3(1-495)$ & \\
\hline
\end{tabular}

$\mathrm{N}=$ number of patients, $\mathrm{SD}=$ standard deviation

*Bleeding complication and/or blood product transfusion

vs. 1.2 days; solid organ: 4.4 vs. 0.6 days; non-cardiac thoracic: 8.9 vs. 2.5 days; general: 4.2 vs. 0.6 days; knee/ hip replacement: 0.2 vs. 0.1 days; reproductive organ: 0.9 vs. 0.04 days; and spine: 1.7 vs. 0.3 days). Lengths of stay differences were unadjusted for covariates.

Table 5 reports unadjusted total hospitalization costs in 2007 US constant dollars. Total costs were higher among patients with a bleeding-related complication and/or blood transfusion versus those without a complication (cardiac: $\$ 43,125$ vs. $\$ 26,073$; vascular: $\$ 40,116$ vs. $\$ 14,288$; non-cardiac thoracic: $\$ 50,081$ vs. $\$ 19,213$; solid organ: $\$ 41,667$ vs. $\$ 16,480$; general: $\$ 28,499$ vs. $\$ 11,937$; knee/hip replacement: $\$ 18,973$ vs. $\$ 14,966$; reproductive organ: $\$ 13,943$ vs. $\$ 6,220$; and spine: $\$ 41,917$ vs. $\$ 20,511)$.

Figure 2 presents mean total hospital costs by surgical subgroup and complication status. Results were adjusted for differences in baseline clinical, demographic, and hospital characteristics between the bleeding-related complication and/or blood product transfusion and no bleeding-related complication and/or blood product transfusion study groups using multivariate ordinary least squares regression modelling techniques. In all surgical subpopulations, the bleeding-related complication and/or blood product transfusion groups had higher total hospital costs versus those without a bleedingrelated complication and/or blood product transfusion $(P<0.0001)$. There were large variations in the incremental differences in mean total costs (bleeding-related complication-no complication) for each of the surgical subgroups (cardiac: $\$ 10,279$; vascular: $\$ 15,123$; non-cardiac thoracic: $\$ 13,473$; solid organ: $\$ 13,210$; general: $\$ 4,354$; knee/hip replacement: $\$ 3,005$; reproductive organ: $\$ 2,805$; and spine: $\$ 17,279$ ). 


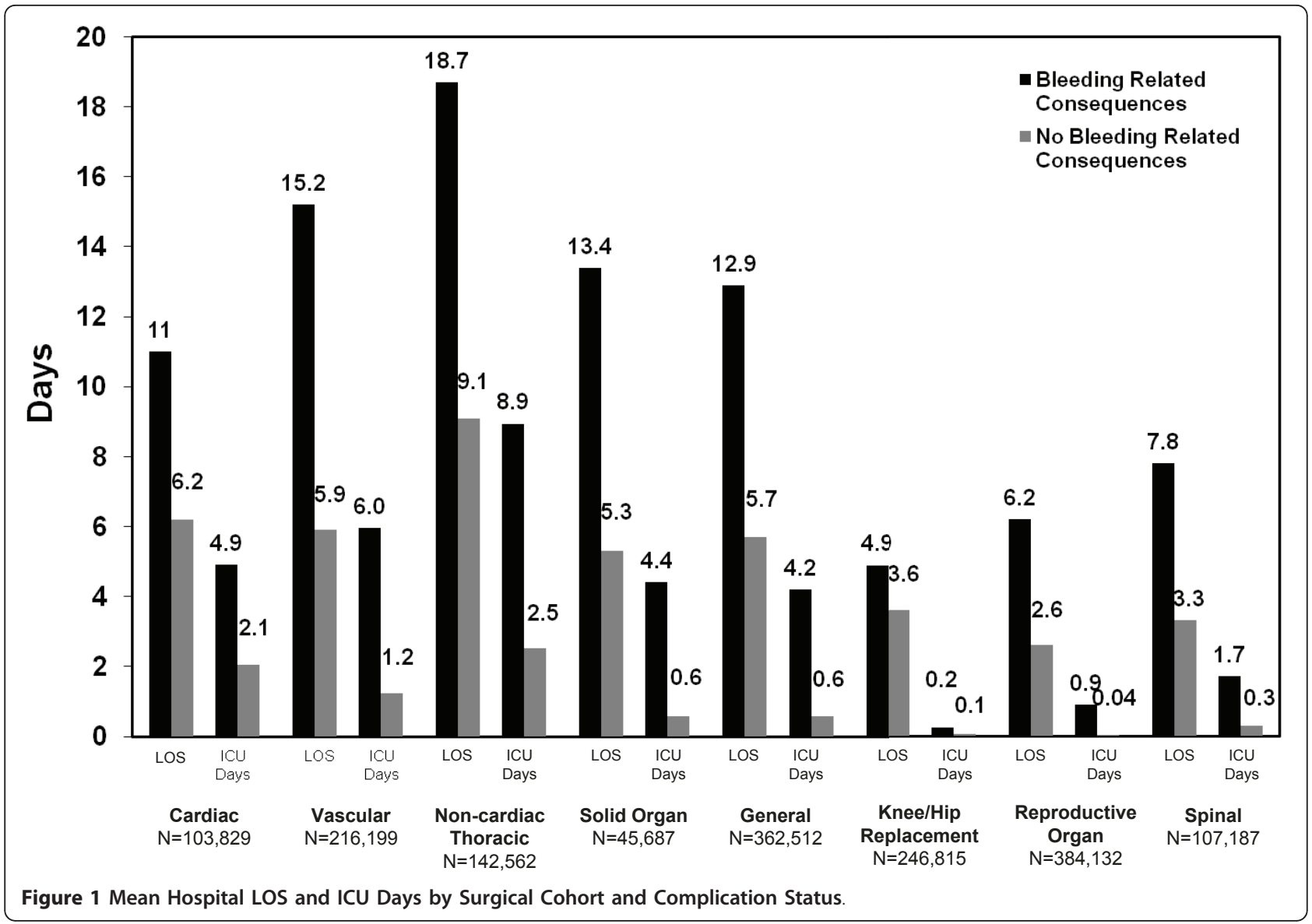

Table 6 reports the ratio of average total costs for patients with bleeding-related complications versus those without bleeding-related complications, adjusted for baseline, clinical characteristics and the effects related to the clustering of patients receiving care from the same hospitals using GEE models. Ordinary least squares model results are also presented for comparison. The ratio of average total costs estimated using GEE models for patients with bleeding complications vs. those without complications ranged from 1.31 to 1.93 for cardiac and vascular surgery, respectively. All average total cost ratios were statistically significant $(P$ $<0.001)$. Cost ratios were similar to those observed using OLS regression with the exception of the ratio for general surgery (1.46 vs. 1.34 for GEE and OLS models, respectively).

\section{Discussion}

We estimated the hospital costs associated with bleeding-related complications in 8 surgical cohorts, including cardiac, vascular, non-cardiac thoracic, solid organ, general surgery, knee/hip replacement, reproductive organ, and spinal surgery, using patient-level data from Premier's PCD. Patients undergoing the surgical procedures of interest during calendar years 2006 and 2007 were identified using ICD-9-CM procedure codes. For each surgical cohort, patients with bleeding-related complications and/or blood product transfusions and those without these events were further delineated using a combination of diagnosis and procedure codes. Mean hospital costs were calculated for each study group after adjusting for differences in baseline demographic and clinical characteristics deemed to have an impact on costs. The incremental difference in adjusted mean costs between patients with a bleeding event and/or blood product transfusion and those without these events was assumed to be an estimate of the hospital costs attributable to having a bleeding event and/or blood product transfusion.

Results of our analyses indicate that bleeding events and/or blood product transfusions were relatively common during the surgical hospitalization and varied according to surgical cohort (general: 27.5\%; cardiac: 47.4\%; solid organ: $28.5 \%$; non-cardiac thoracic: $34.3 \%$; vascular: $31.5 \%$; knee/hip replacement: $29.8 \%$; reproductive organ: $7.5 \%$; and spinal surgery: $15.0 \%)$. Among the types of events identified in this study, blood product transfusions were the most common, occurring in 
Table 5 Unadjusted Total Inpatient Costs (2007 \$US), by Surgical Category and Bleeding-related Complication and/or Blood Product Transfusion Status

\begin{tabular}{|c|c|c|}
\hline Study Measure & Complication* & No Complication \\
\hline \multicolumn{3}{|l|}{ Cardiac } \\
\hline Number of Patients & 49,293 & 54,533 \\
\hline Mean (SD) & $\$ 43,125(\$ 38,283)$ & $\$ 26,073(\$ 22,083)$ \\
\hline Median (Range) & $\begin{array}{c}\$ 33,468(\$ 24,921- \\
\$ 47,466)\end{array}$ & $\$ 22,512(\$ 14,620-\$ 31,365)$ \\
\hline \multicolumn{3}{|l|}{ Vascular } \\
\hline Number of Patients & 68,211 & 147,980 \\
\hline Mean (SD) & $\$ 40,116(\$ 49,461)$ & $\$ 14,288(\$ 19,146)$ \\
\hline Median (Range) & $\begin{array}{c}\$ 24,989(\$ 13,664- \\
\$ 46,806)\end{array}$ & $\$ 9,020(\$ 5,666-\$ 15,952)$ \\
\hline \multicolumn{3}{|l|}{ Non-cardiac thoracic } \\
\hline Number of Patients & 48,854 & 93,699 \\
\hline Mean (SD) & $\$ 50,081(\$ 57,926)$ & $\$ 19,213(\$ 25,388)$ \\
\hline Median (Range) & $\begin{array}{c}\$ 32,695(\$ 16,749- \\
\$ 62,442)\end{array}$ & $\$ 12,334(\$ 7,387-\$ 21,127)$ \\
\hline \multicolumn{3}{|l|}{ Solid organ } \\
\hline Number of Patients & 12,988 & 32,699 \\
\hline Mean (SD) & $\$ 41,667(\$ 49,699)$ & $\$ 16,480(\$ 20,877)$ \\
\hline Median (Range) & $\begin{array}{c}\$ 24,390(\$ 14,772- \\
\$ 47,692)\end{array}$ & $\$ 11,044(\$ 7,890-\$ 17,125)$ \\
\hline \multicolumn{3}{|l|}{ General } \\
\hline Number of Patients & 99,812 & 262,695 \\
\hline Mean (SD) & $\$ 28,499(\$ 42,485)$ & $\$ 11,937(\$ 14,291)$ \\
\hline Median (Range) & $\begin{array}{c}\$ 15,493(\$ 7,980- \\
\$ 31,092)\end{array}$ & $\$ 8,840(\$ 5,934-\$ 13,325)$ \\
\hline \multicolumn{3}{|l|}{ Knee/hip replacement } \\
\hline Number of Patients & 73,795 & 173,020 \\
\hline Mean (SD) & $\$ 18,973(\$ 12,247)$ & $\$ 14,966(\$ 7,075)$ \\
\hline Median (Range) & $\begin{array}{c}\$ 16,256(\$ 13,094- \\
\$ 21,246)\end{array}$ & $\$ 13,742(\$ 11,221-\$ 17,209)$ \\
\hline \multicolumn{3}{|l|}{ Reproductive organ } \\
\hline Number of Patients & 28,951 & 355,180 \\
\hline Mean (SD) & $\$ 13,943(\$ 19,905)$ & $\$ 6,220(\$ 5,009)$ \\
\hline Median (Range) & $\begin{array}{c}\$ 8,849(\$ 6,078- \\
\$ 14,413)\end{array}$ & $\$ 5,262(\$ 4,024-\$ 7,090)$ \\
\hline \multicolumn{3}{|l|}{ Spinal } \\
\hline Number of Patients & 16,068 & 91,117 \\
\hline Mean (SD) & $\$ 41,917(\$ 34,525)$ & $\$ 20,511(\$ 16,114)$ \\
\hline Median (Range) & $\begin{array}{c}\$ 32,813(\$ 22,393- \\
\$ 49,884)\end{array}$ & $\$ 16,557(\$ 10,541-\$ 25,709)$ \\
\hline
\end{tabular}

$\mathrm{SD}=$ standard deviation

*Bleeding complication and/or blood product transfusion

Note: 15 patients were excluded from the economic analysis because their hospital costs exceeded $\$ 1,000,000$

approximately $21.2 \%$ of the patient cohort. Interventions for bleeding control, in contrast, were less common and occurred in only $2.5 \%$ of patients.

In analyses of costs adjusted for disparities in baseline and clinical characteristics, we observed large variations in the incremental difference in mean total costs between patients with a bleeding-related complication and/or blood product transfusion and those without these events for each surgical cohort. Patients with bleeding-related complications and/or blood product transfusions experienced longer hospital lengths of stay and spent more time in the ICU compared to patients without these events.

Studies investigating costs related to bleeding-related complications reveal that they vary widely depending on the surgical cohort. For example, hospital costs attributable to bleeding in trauma patients are reported to be much higher $(\$ 38,628)$ compared to patients undergoing 


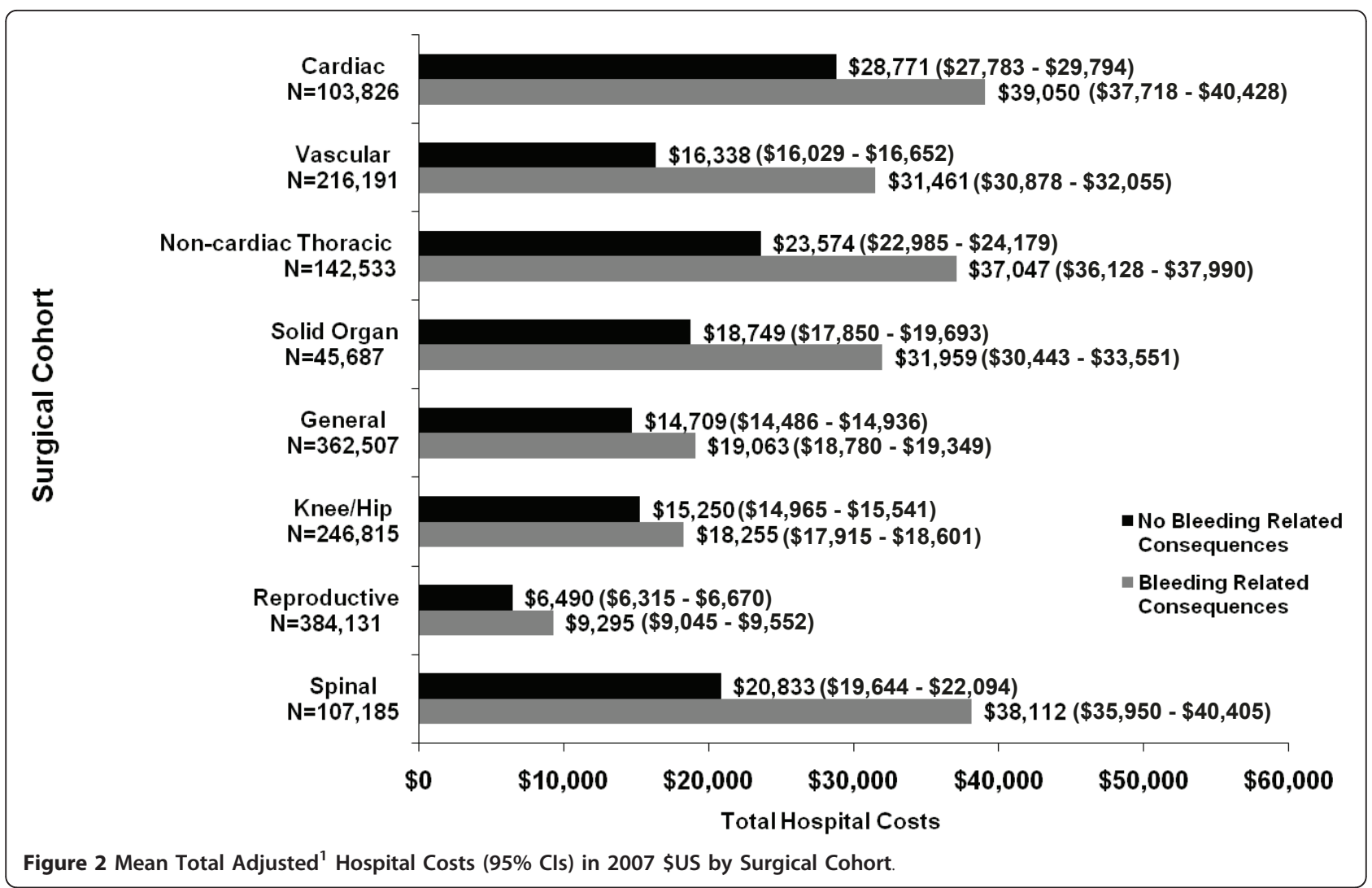

more routine procedures such as PCI $(\$ 5,883)$ and knee and hip surgery $(\$ 7,593)$ [21-23]. Our inpatient cost estimates attributable to bleeding-related complications and/or blood product transfusions ranged from $\$ 2,805$ $\$ 17,279$ for reproductive and spinal surgery, respectively. Considering that the surgical procedures examined in this study were less complex compared to trauma surgery, it makes sense that our estimates are lower than what is previously reported for trauma patients.

This study is subject to several limitations. First, the identification of patients who had a bleeding event and/ or blood product transfusion was based on an algorithm utilizing a combination of ICD-9-CM procedure and diagnosis codes as well as billing charges appearing on

Table 6 Impact of Bleeding Complication Status on Total Hospital Costs: Comparison of GEE and OLS Models

\begin{tabular}{|c|c|c|c|c|}
\hline \multirow[t]{2}{*}{ Surgical Cohort } & \multicolumn{2}{|c|}{ GEE } & \multicolumn{2}{|c|}{ OLS } \\
\hline & Model Estimate $(\mathrm{CI})^{*}$ & $P$-value & Model Estimate $(\mathrm{Cl})^{*}$ & $P$-value \\
\hline Cardiac & $1.31(1.30,1.32)$ & $<0.001$ & $1.36(1.35,1.36)$ & $<0.001$ \\
\hline Vascular & $1.93(1.93,1.95)$ & $<0.001$ & $1.93(1.92,1.95)$ & $<0.001$ \\
\hline Non-cardiac thoracic & $1.60(1.58,1.60)$ & $<0.001$ & $1.57(1.55,1.58)$ & $<0.001$ \\
\hline Solid organ & $1.72(1.68,1.73)$ & $<0.001$ & $1.70(1.68,1.72)$ & $<0.001$ \\
\hline General & $1.46(1.46,1.48)$ & $<0.001$ & $1.34(1.34,1.35)$ & $<0.001$ \\
\hline Knee/hip & $1.22(1.21,1.22)$ & $<0.001$ & $1.20(1.20,1.21)$ & $<0.001$ \\
\hline Reproductive organ & $1.49(1.48,1.51)$ & $<0.001$ & $1.43(1.42,1.43)$ & $<0.001$ \\
\hline Spinal & $1.79(1.77,1.80)$ & $<0.001$ & $1.82(1.80,1.84)$ & $<0.001$ \\
\hline
\end{tabular}

$\mathrm{Cl}=$ confidence interval, GEE = generalized estimating equations, OLS = ordinary least squares

*Ratio of average total costs for patients with bleeding-related complications versus those without complications

Note: 15 patients were excluded from the economic analysis because their hospital costs exceeded $\$ 1,000,000$.

GEE model estimated by gamma log link function with total costs as the dependent variable, adjusted for baseline clinical, demographic, hospital characteristics and clustering on hospital.

OLS model with log total costs as the dependent variable, adjusted for baseline clinical, demographic, and hospital characteristics. 
the hospital record. These coding systems are primarily used for administrative purposes in obtaining reimbursement for the services provided by the hospital. Therefore, we chose to incorporate both bleeding events as well as blood product transfusions in our definition of the study groups. This distinction is important because, with certain surgical procedures, blood product transfusions are routinely administered as part of the surgery and not necessarily because the patient had unexpected bleeding requiring transfusion. Thus, we felt we could not have reliably distinguished one transfusion type from the other. Additionally, it was not possible to differentiate between transfusions occurring as a consequence of the surgery from transfusions that were the result of an underlying or presenting condition such as vascular trauma or ruptured aortic aneurysms if the transfusion occurred on the same day of the surgery as only data pertaining to the day of the transfusion were available. Therefore, it is possible that some patients within the bleeding-event and/or blood product transfusion group had a transfusion that did not occur as a consequence of the surgery and were misclassified. We did not exclude patients with conditions such as vascular trauma or ruptured aortic aneurysms from this analysis in order to facilitate bleeding occurring as a consequence of the surgery. Patients presenting with aortic aneurysms probably would have undergone either open aortic resection with replacement (38.44) or endovascular repair (39.71). The proportion of vascular surgery patients undergoing open aortic resection with replacement was only $1.8 \%$ and patients undergoing endovascular repair were not selected for this analysis. Therefore, we do not expect the inclusion of patients with ruptured aortic aneurysm and the possible misclassification of the bleeding outcome to change results significantly. With respect to vascular trauma, the inclusion of trauma cases that likely had a transfusion as a result of their presenting condition and not the surgical procedure probably introduced some misclassification bias into this study. Since trauma patients have much higher costs relative to patients without trauma, the inclusion of these cases would have had the effect of inflating our bleeding-related complication cost estimates [21]. We suspect that this upward bias is probably minimized by the fact that patients presenting with trauma probably represented only a small percentage of the total patients included in this study. A study from the University of Michigan comparing outcomes between trauma and general surgery patients reported enrolling only 525 patients admitted to the Trauma service in comparison to 54,478 general surgery patients during 2004 [21].

Second, patients were excluded from analyses if they were transferred from another hospital or an unknown source to ensure that complete hospitalization data were available for every patient. We did not feel that we could reliably identify and link multiple hospitalizations for patients transferred to another hospital using the administration codes in the database. As these transferred patients probably had higher costs relative to the current sample of patients, the exclusion of these patients likely had the effect of underestimating the total inpatient episode of care. Furthermore, if more patients with a bleeding-related complication were transferred to other hospitals versus those without bleeding-related complications, the incremental difference in costs between these groups may in fact be larger than what is currently reported.

Third, pediatric patients $<18$ years of age were included in analyses. Although age was included as a covariate in multivariate cost models, one could argue that, because pediatric patients likely underwent different procedures compared to adult patients, separate analyses of these distinct patient groups is warranted. Thus, multivariate cost models examining adult and pediatric patients separately were constructed to provide insight regarding the differences in costs between these two distinct subpopulations. Among adults, patients with bleeding-related complications had higher costs relative to patients without complications (cardiac: $\$ 38,686$ vs. $\$ 28,914$, vascular: $\$ 30,640$ vs. $\$ 16,027$, noncardiac thoracic: $\$ 36,150$ vs. $\$ 23,494$, solid organ: $\$ 31,807$ vs. $\$ 18,878$, general surgery: $\$ 18,880$ vs. $\$ 14,427$, knee/hip replacement: $\$ 18,248$ vs. $\$ 15,247$, reproductive organ: $\$ 9,269$ vs. $\$ 6,493$, and spinal: $\$ 37,978$ vs. $\$ 20,847$ ); results were similar to main analyses and statistically significant. Among the subset of pediatric patients, those with bleeding-related complications had higher costs relative to patients without complications (cardiac: $\$ 58,239$ vs. $\$ 29,514$, vascular: $\$ 113,822$ vs. $\$ 39,506$, non-cardiac thoracic: $\$ 79,898$ vs. $\$ 35,680$, solid organ: $\$ 61,122$ vs. $\$ 28,742$, general surgery: $\$ 104,505$ vs. $\$ 37,316$, reproductive organ: $\$ 34,703$ vs. $\$ 16,999$, and spinal: $\$ 54,369$ vs. $\$ 31,984)$. A multivariate model was not created for pediatric knee/hip surgery patients because of the small sample size relative to the number of parameters in the model. Overall, costs in patients $<18$ years of age were higher compared to the adult population. The incremental difference in costs between patients with bleeding-related consequences and those without bleeding-related consequences was also higher in pediatric patients compared to adults.

Fourth, our analyses of costs were adjusted for differences in baseline clinical and demographic characteristics using multivariate ordinary least squares regression with Duan's smearing back retransformation. The approach used for retransformation should be 
dependent upon the nature of the error term on the transformed scale [24]. Since the distribution of the error term is usually unknown, reliance on the assumption of normality or homoskedasticity can lead to inconsistent estimates [24]. Therefore, we also examined the sensitivity of results to retransformation using subgroup-specific smearing factors as proposed by Manning [25]. Using this alternate retransformation method, cost estimates for patients with bleeding-related complications remained statistically significantly higher versus those without complications. In fact, the incremental differences in mean total costs (bleeding-related complication - no complication) for each of the surgical subgroups were higher compared to the main study results (cardiac: $\$ 17,055$, vascular: $\$ 25,795$, non-cardiac thoracic: $\$ 30,963$, solid organ: $\$ 25,153$, general: $\$ 16,567$, knee/hip replacement: $\$ 4,009$, reproductive organ: $\$ 7,707$, and spinal surgery: $\$ 21,326$ ).

It should be noted that a small percentage of patients (approximately 5\%) were included in more than one study subgroup because they were operated on in surgical sites spanning multiple surgical categories during the index hospitalization. A sensitivity analysis excluding these patients was conducted to examine the effect they might have had on cost results. Results show that their effect was minimal as the incremental difference in mean total costs (bleeding-related complication - no complication) were similar to the main analyses for each of the surgical subgroups (cardiac: $\$ 10,391$, vascular: $\$ 14,639$, non-cardiac thoracic: $\$ 12,366$, solid organ: $\$ 12,434$, general: $\$ 3,974$, knee/hip replacement: $\$ 3,148$, reproductive organ: $\$ 3,266$, and spinal surgery: $\$ 17,448$ ).

All baseline and clinical characteristics deemed to have an impact on total hospital costs were included in our models. Nevertheless, we could not control for certain covariates known to affect costs because this information was not available in the PCD. These covariates included sociodemographic parameters such as smoking status and whether the patient was living alone. Although we identified patients who were obese using ICD-9-CM diagnosis codes (278.00-278.02), to the best of our knowledge it is unknown to what extent these codes can be used to accurately identify individuals who are obese. Additionally, data on certain chronic comorbid conditions were likely underestimated. Comorbidity data were only available if a patient was treated for the condition during the index hospitalization or if a patient was treated in a prior admission to a hospital within Premier's data capture network. Because data on outpatient physician visits are not included in the PCD, for some comorbidities including thrombocytopenia, MI, hypertension, non-MI coronary disease, renal disease, congestive heart failure, and deep vein thrombosis it was impossible to determine whether the condition was chronic and unrelated to the index surgical hospitalization or occurred as an adverse event related to the surgery or bleeding complication. Many patients also did not have prior hospitalization data, therefore many comorbidities were identified within the index surgical hospitalization. As a result, the assumption of independence between covariates in our multivariate analyses may have been violated if, for example, certain conditions such as MI or renal failure developed as a result of the bleeding complication and were not actually pre-existing conditions. As a result regression models could have produced inaccurate estimates of regression coefficients, variability, and $P$-values. Therefore, multivariate cost analyses were also run using only the conditions that were deemed to be obvious chronic conditions identifiable in the database. These conditions included diabetes, obesity, COPD, cancer, and cirrhosis. The incremental cost per hospitalization associated with bleeding-related complications was similar to the main study results $(\$ 15,931$ for vascular, $\$ 15,410$ for solid organ, $\$ 14,653$ for non-cardiac thoracic, $\$ 11,715$ for cardiac, $\$ 5,114$ for general, $\$ 3,119$ for knee/hip replacement, $\$ 2,961$ for reproductive organ, and $\$ 17,707$ for spinal surgery) and the difference in costs among patients with bleeding-related complications and those without complications was statistically significant for every surgical cohort $(P<0.001)$. We also did not control for conditions such as coagulopathy, anemia, or the use of bone marrow suppressants. If a higher percentage of patients in the bleeding complication group had these prior conditions or procedures, our cost estimates related to bleeding complications may have been overstated especially if a prior hospitalization for these conditions or procedures was associated with higher costs during the study index surgical hospitalization. Finally, information relating to the severity of the comorbid condition was not available. As severity could have an impact on hospital costs, the inability to control for severity is another limitation of our analysis.

\section{Conclusions}

These analyses offer insight into the magnitude of differences in costs between patients with and without bleeding-related complications and/or blood product transfusions among hospital-based procedures in 7 specialties. The optimal management of bleeding is an important goal, as these complications are associated with increased morbidity and mortality in a variety of different surgical populations [2-4,7]. Given the high costs associated with managing patients with bleedingrelated complications and/or blood product transfusions, additional comprehensive approaches should be evaluated or developed to optimize intra-operative 
bleeding management, including product and technique strategies.

\section{Key Messages}

- In every surgical cohort examined, patients with bleeding-related complications and/or blood product transfusions had longer lengths of stays and higher total hospital costs.

- There were large variations in the incremental differences in total hospital costs between bleedingrelated complication and no complication cohorts for each of the surgical subgroups (cardiac: $\$ 10,279$; vascular: $\$ 15,123$; non-cardiac thoracic: $\$ 13,473$; solid organ: $\$ 13,210$; general: $\$ 4,354$; knee/hip replacement: $\$ 3,005$; reproductive organ: $\$ 2,805$; and spine: $\$ 17,279)$.

- Patients with bleeding-related complications and/or blood product transfusions spent, on average, approximately 2.7 more days in an ICU compared to patients without bleeding-related complications and blood product transfusions (overall: 3.3 vs. 0.5 days).

- Given the high costs associated with managing patients with bleeding-related complications and/or blood product transfusions, additional comprehensive approaches should be evaluated or developed to optimize intra-operative bleeding management, including product and technique strategies.

\section{Funding}

Funding for this study was provided by Ethicon, Inc.

\section{List of Abbreviations}

COPD: Chronic obstructive pulmonary disease; ICU: Intensive care unit; LOS. Length of stay; MI: Myocardial infarction; PCD: Premier's Perspective ${ }^{\mathrm{TM}}$ Comparative Database; PCI: Percutaneous coronary intervention; US: United States.

\section{Author details}

${ }^{1}$ United BioSource Corporation, Montréal, QC, Canada. ${ }^{2}$ Ethicon Inc., Somerville, NJ, USA. ${ }^{3}$ Xcenda, Palm Harbor, FL, USA. ${ }^{4}$ United BioSource Corporation, Lexington, MA, USA.

\section{Authors' contributions}

MES participated in the design and coordination of the study, analysis and interpretation of the data, and the drafting of the manuscript. KM created the study analytic file from the raw data, carried out the statistical analysis, and participated in the revision of the manuscript. XY, MFTR, and JH participated in the design of the study, analysis and interpretation of the data, and revision of the manuscript. MS and MR participated in the design of the study and analysis and interpretation of the data. MFTR and MR were also involved in study conception. All authors read and approved the final manuscript.

\section{Competing interests}

Michael E. Stokes, Katie Mercaldi, and Matthew W. Reynolds are full-time employees of United BioSource Corporation. United BioSource Corporation received funding from Ethicon, Inc. for the conduct of this study and drafting of the manuscript. Manan Shah is an employee of Excenda, who received funding from Ethicon, Inc. for study consultation. Xin Ye, Marcia FT
Rupnow, and Jeffrey Hammond are full-time employees of Ethicon, Inc. and receive a salary from Ethicon.

United BioSource Corporation is a global scientific and medical affairs organization that partners with life science companies to help generate realworld evidence of product effectiveness, safety, and value to assist health care decisions and enhance patient care. Ethicon Inc. is a global medical device company with major products covering wound closure; hernia repair; biosurgery; women's health, aesthetic medicine and ENT. It develops, manufactures and markets a variety of products designed to achieve adjunctive hemostasis. Excenda is a full-service consultancy and managed markets agency that helps manufacturers identify, demonstrate, and deliver their brand's value proposition to healthcare stakeholders. UBC and Excenda are independent consulting firms that were contacted by Ethicon to perform this study.

Received: 1 April 2010 Accepted: 31 May 2011 Published: 31 May 2011

\section{References}

1. Shander A: Financial and clinical outcomes associated with surgical bleeding complications. Surgery 2007, 142(4 Suppl):S20-25.

2. Rady MY, Ryan T, Starr NJ: Perioperative determinants of morbidity and mortality in elderly patients undergoing cardiac surgery. Crit Care Med 1998, 26(2):225-235.

3. Marietta M, Facchini L, Pedrazzi P, Busani S, Torelli G: Pathophysiology of bleeding in surgery. Transplant Proc 2006, 38(3):812-814.

4. Spahn DR, Cerny V, Coats TJ, Duranteau J, Fernandez-Mondejar E, Gordini G, Stahel PF, Hunt BJ, Komadina R, Neugebauer E, et al: Management of bleeding following major trauma: a European guideline. Crit Care 2007, 11(1):R17.

5. Maluenda G, Lemesle G, Ben-Dor I, Collins SD, Syed Al, Li Y, Torguson R, Kaneshige K, Xue Z, Suddath WO, et al: Value of blood transfusion in patients with a blood hematocrit of $24 \%$ to $30 \%$ after percutaneous coronary intervention. Am J Cardiol 2009, 104(8):1069-1073.

6. O'Neill WW: Risk of bleeding after elective percutaneous coronary intervention. N Engl J Med 2006, 355(10):1058-1060.

7. Kinnaird TD, Stabile E, Mintz GS, Lee CW, Canos DA, Gevorkian N, Pinnow EE, Kent KM, Pichard AD, Satler LF, et al: Incidence, predictors, and prognostic implications of bleeding and blood transfusion following percutaneous coronary interventions. Am J Cardiol 2003, 92(8):930-935.

8. Taylor RW, O'Brien J, Trottier SJ, Manganaro L, Cytron M, Lesko MF, Arnzen K, Cappadoro C, Fu M, Plisco MS, et al: Red blood cell transfusions and nosocomial infections in critically ill patients. Crit Care Med 2006, 34(9):2302-2308, quiz 2309.

9. Rogers MA, Blumberg N, Saint S, Langa KM, Nallamothu BK: Hospital variation in transfusion and infection after cardiac surgery: a cohort study. BMC Med 2009, 7:37.

10. Spiess BD: Blood transfusion: the silent epidemic. Ann Thorac Surg 2001, 72(5):S1832-1837.

11. Toy P, Lowell C: TRALI-definition, mechanisms, incidence and clinical relevance. Best Pract Res Clin Anaesthesiol 2007, 21(2):183-193.

12. Robb WJ: Massive transfusion in trauma. AACN Clin Issues 1999, 10(1):69-84, quiz 138-140.

13. Engoren M, Mitchell E, Perring P, Sferra J: The effect of erythrocyte blood transfusions on survival after surgery for hip fracture. J Trauma 2008, 65(6):1411-1415.

14. DeFrances C, Lucas C, Buie V, Golosinskiy A: 2006 National Hospital Discharge Survey. In National Health Statistics Reports. Volume 5. Hyattsville, MD: National Center for Health Statistics; 2008.

15. Premier Inc: Premier Perspective ${ }^{\mathrm{TM}}$. Charlotte, NC: Premier Inc; 2009.

16. Yank V, Tuohy CV, Logan AC, Bravata DM, Staudenmayer K, Eisenhut R, Sundaram V, McMahon D, Stave CD, Zehnder JL, et al: Comparative Effectiveness of Recombinant Factor Vlla for Off-Label Indications vs. Usual Care. Comparative Effectiveness Review No 21 Rockville, MD: Agency for Healthcare Research and Quality; 2010, \#290-02-0017 S-UE-bPCuCN.

17. Facts and Figures 2007 - Table of Contents. Healthcare Cost and Utilization Project (HCUP) September 2009. [http://www.hcup-us.ahrq. gov/reports/factsandfigures/2007/TOC_2007.jsp].

18. Regulations and Ethical Guidelines: Title 45 Public Welfare Department of Health and Human Services, Part 46 Protection of Human Subjects. Revised June 23, 2005. [http://ohsr.od.nih.gov/guidelines/45cfr46.html]. 
19. Blanchette CM, Wang PF, Joshi AV, Asmussen M, Saunders W, Kruse P: Cost and utilization of blood transfusion associated with spinal surgeries in the United States. Eur Spine J 2007, 16(3):353-363.

20. Duan N: Smearing estimate: a nonparametric retransformation method. $f$ Am Stat Assoc 1983, 78(383):605-610.

21. Hemmila MR, Jakubus JL, Maggio PM, Wahl WL, Dimick JB, Campbell DA Jr, Taheri PA: Real money: complications and hospital costs in trauma patients. Surgery 2008, 144(2):307-316.

22. Jacobson KM, Hall Long K, McMurtry EK, Naessens JM, Rihal CS: The economic burden of complications during percutaneous coronary intervention. Qual Saf Health Care 2007, 16(2):154-159.

23. Vera-Llonch M, Hagiwara M, Oster G: Clinical and economic consequences of bleeding following major orthopedic surgery. Thromb Res 2006, 117(5):569-577.

24. Mihaylova B, Briggs A, O'Hagan A, Thompson SG: Review of statistical methods for analysing healthcare resources and costs. Health Econ 2010.

25. Manning WG: The logged dependent variable, heteroscedasticity, and the retransformation problem. J Health Econ 1998, 17(3):283-295.

\section{Pre-publication history}

The pre-publication history for this paper can be accessed here: http://www.biomedcentral.com/1472-6963/11/135/prepub

doi:10.1186/1472-6963-11-135

Cite this article as: Stokes et al:. Impact of bleeding-related complications and/or blood product transfusions on hospital costs in inpatient surgical patients. BMC Health Services Research 2011 11:135.

\section{Submit your next manuscript to BioMed Central and take full advantage of:}

- Convenient online submission

- Thorough peer review

- No space constraints or color figure charges

- Immediate publication on acceptance

- Inclusion in PubMed, CAS, Scopus and Google Scholar

- Research which is freely available for redistribution

Submit your manuscript at www.biomedcentral.com/submit 